\section{A program to perform Thurstone's method of successive integrals}

\author{
KARL HUNT \\ University of Tennessee at Chattanooga \\ Chattanooga, Tennessee 37401
}

METHSUCI (METHod of SUCcessive Integrals) is a program which performs Thurstone's method of successive integrals for up to 300 stimuli, following procedures described by Guilford (1954; Pp. 237-241). The first step is to determine the proportion of ratings that fall in each rating category for each stimulus. These proportions are then used to obtain mean values for the rating categories, stated in terms of unit-normal standard deviations. Next, the category means are averaged across stimuli and used to estimate overall mean differences between adjacent rating categories. These differences are used to construct a common scale with interval scale characteristics. Each category on the scale is given a value based on its distance from the mean of the first category, as indicated by the differences. The first category's mean, of course, can be set equal to any arbitrary value; the value used by METHSUCI is 1.

Values for each stimulus are then calculated by the following formula:

$$
\mathrm{S}=\Sigma \mathrm{p}_{\mathbf{i}} C_{\mathrm{i}}
$$

where $S$ is the stimulus value, $p_{i}$ is the proportion of ratings in category $i$ for that stimulus, and $C_{i}$ is the common-scale category value. METHSUCI also determines a standard deviation for each stimulus:

$$
\mathrm{SD}=\left[\Sigma \mathrm{p}_{\mathrm{i}} \mathrm{C}_{\mathrm{i}}^{2}-\left(\Sigma \mathrm{p}_{\mathrm{i}} \mathrm{C}_{\mathrm{i}}\right)^{2}\right]^{1 / 2}
$$

Input. The card input to the program must consist of a parameter card indicating (a) the number of stimuli (up to 300 ), (b) the number of rating categories (up to seven), and (c) the number of different stimulus orders (up to ten). The last parameter allows the user to utilize the data from several lists made up of different presentation orders without having to re-sort the data before entering them into the computer.

A second parameter card provides the user the options of printing some of the intermediate steps in the data analysis, along with different stimulus values obtained by variations in the basic procedure as described in the output section. Following the second parameter card are cards that indicate the order of stimuli for each list. These are followed by the data cards. The latter contain the ratings generated by the subjects for the stimuli.

Output. As indicated by the second parameter card, the program provides a printout of (a) cumulative proportions for each rating category of each stimulus, (b) common-scale category values for each of two groups in the same data set (e.g., females and males), and for the groups combined, (c) stimulus values for each of the scales in $b$, along with standard deviations, and (d) standardized stimulus values, i.e., the stimulus values divided by their respective standard deviations.

Computer and language. The program is written in FORTRAN IV and has been run on an IBM 360 under level G and $\mathrm{H}$ compilers. The memory requirements are $110 \mathrm{~K}$ for compilation under the level $\mathrm{G}$ compiler, and $160 \mathrm{~K}$ for running the program. The latter could be reduced by changing array dimensions if fewer stimuli/categories or list orders are used.

Availability. A listing and documentation are available free from the author at the Department of Psychology, University of Tennessee at Chattanooga, Chattanooga, Tennessee 37401.

\section{REFERENCES}

Guilford, J. P. Psychometric methods. (2nd ed.) New York: McGraw-Hill, 1954.
Behavior Research Methods \& Instrumentation 1975, Vol. 7 (6), 576

\section{SUBSIM: A subject simulation program for on-line conformity research}

\section{STEVEN R. SELIGMAN and DAVID J. STANG Queens College of the City University of New York Flushing, New York 11367}

The Crutchfield apparatus for studying conformity behavior allows the experimenter to lead subjects to believe they are seeing each other's responses, when in fact the answers, indicated by illuminated lights on a panel, are provided by the experimenters. The apparatus is clever and effective, but it is also very expensive and tedious to construct, cumbersome to store, and requires running a fixed number of subjects at once. These considerations generally mean that groups of only 4 or 5 can be run. SUBSIM enables the experimenters to run one subject at a time, on-line; he can simulate the responses of up to nine other subjects, presumably seated at other terminals.

Interaction with SUBSIM begins when SUBSIM presents instructions to the subject and the cover story (in this case an impression formation task), and assigns the subject to a station and response position. SUBSIM then asks each subject for his (her) name, age, sex, and identification number, providing experimenter-supplied information for the simulated subjects. Random delays between the request for the information and the computer-supplied simulated responses add to the credibility of the situation. SUBSIM then presents up to 40 questions, providing simulated answers to each, and stores the real subject's responses and response latencies in a file labeled with identification numbers. Various checks in SUBSIM prohibit inappropriate responses and instruct the subject to enter responses in the correct form.

SUBSIM is easily modified to vary the number and descriptions of simulated subjects, the subject's response position, the cover story, and the number and nature of the items. Debriefing is left to the experimenters.

Language. SUBSIM was developed on a Xerox Sigma 7 and is written in Xerox Extended FORTRAN IV with 9 assembly language subroutines.

Availability. Documented copies of SUBSIM are available free of charge from Steven Seligman, 98-21 65th Avenue, Rego Park, New York 11374, or David Stang, Department of Psychology, Queens College, Flushing, New York 11367. 